\title{
SEARCH FOR A "GRAVITOID" THEORY
}

\author{
J. A. Nieto ${ }^{1}$ and M. C. Marín \\ Departamento de Investigación en Física de la Universidad de Sonora, \\ 83190, Hermosillo Sonora, México \\ and \\ Facultad de Ciencias Físico-Matemáticas de la Universidad Autónoma \\ de Sinaloa, 80010, Culiacán Sinaloa, México ${ }^{2}$
}

\begin{abstract}
By combining the concepts of graviton and matroid, we outline a new gravitational theory which we call gravitoid theory. The idea of this theory emerged as an attempt to link the mathematical structure of matroid theory with M-theory. Our observations are essentially based on the formulation of matroid bundle due to MacPherson and Anderson-Davis. Also, by considering the oriented matroid theory, we add new observations about the link between the Fano matroid and $D=11$ supergravity which was discussed in some of our recent papers. In particular we find a connection between the affine matroid $A G(3,2)$ and the $G_{2}$-symmetry of $D=11$ supergravity.
\end{abstract}

Pacs numbers: 04.60.-m, 04.65.+e, 11.15.-q, 11.30.Ly

February, 2003

\footnotetext{
${ }^{1}$ nieto@uas.uasnet.mx

${ }^{2}$ permanent address
} 


\section{INTRODUCTION}

It is known that the theory called M-theory ${ }^{1-3}$ was suggested by duality symmetries interrelating the five known superstring theories ${ }^{4}$ in $9+1$ dimensions (i.e., nine space and one time), Type I, Type IIA, Type IIB, Heterotic $\mathrm{SO}(32)$ and Heterotic $\mathrm{E}_{8} \times \mathrm{E}_{8}$. If the strong/weak coupling duality transformations allow to relate these superstrings theories then it is no longer appropriate to see them as distinct theories, but rather as different manifestations of an underlying unique theory: M-theory. Roughly speaking, we can say that duality implies M-theory.

It turns out that in an apparent complete different area and in another time an analogue conclusion led Whitney ${ }^{5}$ to the discovery of the concept of matroid in 1935. In effect, it is known that not all of the graphs have an associated dual graph. If one insists on extending the duality property to any graph, not only for plane graphs, one is inevitably led to the mathematical structure of matroids. From this point of view, we can also say that duality implies a matroid scenario.

Now, the arising question is whether these two conclusions are not a coincidence. But, why to ask, first of all, for a coincidence if M-theory and matroid structure appear, at first sight, as two totally different formalisms. This scenario seems to be similar to the case of the two apparently unrelated subjects: Newton's gravitational theory and the mathematics of Riemann geometry. The equivalence principle, among other things, suggested to look for a mathematical structure beyond the Euclidean geometry used in Newton's gravitational theory. It is well known that in 1915 it was discovered that the use of the Riemann geometry makes possible the accomplishment of such a quest. Similarly, a duality principle in M-theory may suggest the search of a singular mathematical structure beyond the mathematical formalism used in string theory. In three previous works ${ }^{6-8}$ we provided enough evidence to prove that a duality principle in M-theory may have a mathematical support in the well-developed formalism of matroid-theory ${ }^{9-13}$. In fact, in these references it was shown that Fano matroid and the matroid-Tutte polynomial are connected to $D=11$ Supergravity, Chern-Simons theory and string theory, which are essential parts of M-Theory.

Further, the connection between matroid theory and $D=11$ supergravity

may naturally lead to the need of a mathematical structure that combines matroids and fiber bundles. The reason for this is that in order to have a 
realistic four dimensional theory it is necessary to compactify seven dimensions out of the eleven space-like dimensions of $D=11$ supergravity. The traditional method to achieve such a compactification is via the Kaluza-Klein mechanism. But, as it is well known, this mechanism is closely related to the mathematical structure of fiber bundles.

Fortunately, the concept of matroid bundle has already been developed by the mathematicians. In fact, in 1993 MacPherson $^{14}$ defined for the first time matroid bundles in connection with combinatorial differential manifolds. Since then the matroid bundles structure has been refined and developed by Anderson and Davis, ${ }^{15,25}$ and others ${ }^{16-17}$. Roughly speaking, matroid bundles are the combinatorial analogue of vector bundles. The central idea in matroid bundles is to allow oriented matroids ${ }^{18}$ to play the role of tangent spaces or, more generally, vector spaces.

Now, vector bundles provide an important tool in the formulation of the four dimensional gravitational theory contained in $D=11$ supergravity. Therefore, one should expect that matroid bundles may lead to a new gravitational structure. We call this expected new gravitational object the "gravitoid" because it combines the concepts of graviton and matroid. Our conjecture is that the gravitoid theory should play a crucial role not only in $D=11$ supergravity but also in M-theory itself. Since every matroid has an associated unique dual it is reasonable to expect that the gravitoid should have an associated unique dual gravitoid and this fact should lead to a dual invariant partition function for the gravitoid.

The plan of this work is as follows: In section II, we briefly review oriented matroid theory. In section III, we closely follow the Ref. 6 adding new information about the connection between matroid theory and $D=11$ supergravity. In particular, we discuss a link between the affine matroid $A G(3,2)$ and $G_{2}$-symmetry of $D=11$ supergravity via self-duality. In section IV, we introduce the concept of matroid bundle and develop the gravitoid theory. Finally, in section V, we remark the importance of gravitoid theory and matroid bundles for further research and make some final comments.

\section{A BRIEF REVIEW OF ORIENTED MATROIDS}

Matroid theory ${ }^{9-13}$ arose in 1935 from the abstract properties of vector spaces and graph theory. Whitney ${ }^{5}$ seems to be the first mathematician to have noticed such properties. Looking at Whitney's previous work ${ }^{19}$ on 
non-separable graph theory, one wonders if the lack of duality on nonplanar graphs, such as the complete graph $K_{5}$ and the bipartite graph $K_{3,3}$, provided his main motivation to introduce such abstract properties. Indeed, as it is known, in graph theory only planar graphs have an associated dual graph. As the Kuratowski's theorem assures, the complete graph $K_{5}$ and the bipartite graph $K_{3,3}$, which are nonplanar, do not have a corresponding dual graph. On the other hand, matroid theory has the attractive feature that every matroid has an associated unique dual matroid. In particular, the matroid associated to $K_{5}$, let us say $M\left(K_{5}\right)$, has the dual matroid $M^{*}\left(K_{5}\right)$, but $M^{*}\left(K_{5}\right)$ turns out to be not representable by means of a graph.

In connection with vector spaces, the matroid duality is realized by the orthogonality of matrices. But the notion of orthogonality in vector spaces is extended by matroid theory in the sense that not all matroids are realizable by matrices. In fact, there are matroids, such as the non-Pappus matroid, ${ }^{9}$ that are not realizable by a subset of a vector space or by matrices.

A natural generalization of graph theory is provided by oriented graph theory. In this generalized case, the edges of a graph are labeled with a plus or minus sign. Similarly, a natural generalization of matroid theory is the oriented matroid theory. The origin of oriented matroid theory can be traced back to the Gutierrez-Novoa's work ${ }^{20}$ on signed bases, in 1965 . However, it seems that the formal axiomatic notion of orientability for matroids was not proposed before 1978 (see Ref. 18 for details). One of the attractive features of oriented matroids is that they capture the sign properties of vector spaces without losing the duality properties of the underlying nonoriented matroids. At present, oriented matroid theory is a well-developed mathematical structure in combinatorics with a very interesting connection in differential topology, as we shall discuss in section IV.

An oriented matroid $\mathcal{M}$ is a pair $(E, \mathcal{C})$, where $E$ is a non-empty finite set, and $\mathcal{C}$ is a non-empty collection of subsets of $E$ (called signed circuits) satisfying the following properties:

$(\mathcal{C} i)$ no circuit properly contains another circuit,

$(\mathcal{C}$ ii $)$ if $\mathcal{C}_{1}$ and $\mathcal{C}_{2}$ are two distinct signed circuits, $\mathcal{C}_{1} \neq-\mathcal{C}_{2}$, and $c \in$ $\mathcal{C}_{1}^{+} \cap \mathcal{C}_{2}^{-}$then there exists a third circuit $\mathcal{C}_{3} \in \mathcal{C}$ with $\mathcal{C}_{3}^{+} \subseteq\left(\mathcal{C}_{1}^{+} \cap \mathcal{C}_{2}^{+}\right) \backslash\{c\}$ and $\mathcal{C}_{3}^{-} \subseteq\left(\mathcal{C}_{1}^{-} \cap \mathcal{C}_{2}^{-}\right) \backslash\{c\}$

An alternative, but equivalent, way to define an oriented matroid is as follows: An oriented matroid $\mathcal{M}$ is a pair $(E, \chi)$, where $E$ is a non-empty finite set and $\chi$ (called chirotope) is a mapping $E^{r} \rightarrow\{-1,0,1\}$, with $r$ the rank on $E$, satisfying the following properties. 
$(\chi i) \chi$ is not identically zero,

$(\chi i i) \chi$ is alternating,

( $\chi$ iii) for all $x_{1}, x_{2}, \ldots, x_{r}$ and $y_{1}, y_{2}, \ldots, y_{r}$ such that

$$
\chi\left(x_{1}, x_{2}, \ldots, x_{r}\right) \chi\left(y_{1}, y_{2}, \ldots, y_{r}\right) \neq 0,
$$

there exists an $i \in\{1,2, \ldots, r\}$ such that

$\chi\left(y_{i}, x_{2}, \ldots, x_{r}\right) \chi\left(y_{1}, y_{2}, \ldots, y_{i-1}, x_{1}, y_{i+1}, \ldots, y_{r}\right)=\chi\left(x_{1}, x_{2}, \ldots, x_{r}\right) \chi\left(y_{1}, y_{2}, \ldots, y_{r}\right)$

For a vector configuration the chirotope $\chi$ can be identified as

$$
\chi\left(i_{1}, \ldots, i_{r}\right) \equiv \operatorname{sign} \operatorname{det}\left(v_{i_{1}}, \ldots, v_{i_{r}}\right) \in\{-1,0,1\}
$$

and in this case (2) becomes connected with the Grassmann-Plucker relation.

It can be proved that the definition of the underlying matroid $M$ of $\mathcal{M}$ follows from the chirotope definition for oriented matroids. In fact, from the chirotope definition it follows that if $\mathcal{B}$ is the set of $r$-subsets of $E$ such that

$$
\chi\left(x_{1}, x_{2}, \ldots, x_{r}\right) \neq 0,
$$

for some ordering of $\left(x_{1}, x_{2}, \ldots, x_{r}\right)$ of $\mathcal{B}$, then $\mathcal{B}$ is the set of bases of the matroid $M$. Formally, the definition of $M$ in terms of the bases is as follows:

A matroid $M$ is a pair $(E, \mathcal{B})$, where $E$ is a non-empty finite set and $\mathcal{B}$ is a non-empty collection of subsets of $E$ (called bases) satisfying the following properties:

$(\mathcal{B} i)$ no base properly contains another base;

$(\mathcal{B}$ ii $)$ if $B_{1}$ and $B_{2}$ are bases and if $b$ is any element of $B_{1}$, then there is an element $g$ of $B_{2}$ with the property that $\left(B_{1}-\{b\}\right) \cup\{g\}$ is also a base.

The dual of $M$, denoted by $M^{*}$, is defined as a pair $\left(E, \mathcal{B}^{*}\right)$, where $\mathcal{B}^{*}$ is a non-empty collection of subsets of $E$ formed with the complements of the bases of $M$. An immediate consequence of this definition is that every matroid has a dual and this dual is unique. It also follows that the doubledual $M^{* *}$ is equal to $M$.

The definition of the corresponding dual oriented matroid $\mathcal{M}^{*}$ is straightforward. One introduces the dual chirotope $\chi^{*}$ such that

$$
\chi^{*}: E^{n-r} \rightarrow\{-1,0,1\}
$$


and

$$
\left(x_{1}, x_{2}, \ldots, x_{n-r}\right) \rightarrow \chi\left(x_{1}^{\prime}, x_{2}^{\prime}, \ldots, x_{r}^{\prime}\right) \operatorname{sign}\left(x_{1}, x_{2}, \ldots, x_{n-r}, x_{1}^{\prime}, x_{2}^{\prime}, \ldots, x_{r}^{\prime}\right),
$$

where $\left(x_{1}^{\prime}, x_{2}^{\prime}, \ldots, x_{r}^{\prime}\right)$ means some permutation of $E \backslash\left(x_{1}, x_{2}, \ldots, x_{n-r}\right)$ and

$$
\operatorname{sign}\left(x_{1}, x_{2}, \ldots, x_{n-r}, x_{1}^{\prime}, x_{2}^{\prime}, \ldots, x_{r}^{\prime}\right)
$$

is the parity of the number of inversions of $(1,2, \ldots, n)$. It is not difficult to see that, as in the case of ordinary matroids, every oriented matroid $\mathcal{M}(E, \chi)$ has an associated unique dual $\mathcal{M}^{*}\left(E, \chi^{*}\right)$. Furthermore, it is found that $\mathcal{M}^{* *}=$ $\mathcal{M}$.

Another interesting and important result is that

$$
\left(\mathcal{M}_{1} \oplus \mathcal{M}_{2}\right)^{*}=\mathcal{M}_{1}^{*} \oplus \mathcal{M}_{2}^{*},
$$

where $\mathcal{M}_{1} \oplus \mathcal{M}_{2}$ is the direct sum of two connected oriented matroids $\mathcal{M}_{1}$ and $\mathcal{M}_{2}$.

\section{ORIENTED MATROIDS, OCTONIONS, $G_{2}$-SYMMETRY AND $D=11$ SUPERGRAVITY}

In this section, we shall extend the discussion presented in Ref.6, 7 and 8 on the connection between matroid theory and $D=11$ supergravity. In particular, we show that the complementary relation in seven dimensions between the Fano matroid $F_{7}$ and octonions can be extended to eight dimensions by means of the affine matroid $A G(3,2)$. We also prove that the selfduality condition on the octonionic structure in eight dimensions (necessary to account for the $G_{2}$-symmetry in $D=11$ supergravity) is in concordance with the self-duality of $A G(3,2)$. At the same time, the idea of this section is to prepare the motivation and notation to consider matroid bundles and the gravitoid theory in the next section.

It is known that in order to get a realistic four dimensional theory from $D=11$ supergravity it is necessary to compactify seven space-like dimensions out of the eleven dimensions. At this respect, the Englert's compactification ${ }^{21}$ of $D=11$ supergravity provides us with one of the most attractive solutions. In this case, the $D=11$ manifold $B^{11}$ is compactified in the form $B^{4} \times S^{7}$, 
where $B^{4}$ is a four dimensional space-time manifold and $S^{7}$ is the seven dimensional sphere. Such a solution turns out to be connected to the parallelizable properties of $S^{7}$, which in turn are related to octonions. ${ }^{22}$ Moreover, in the Englert's mechanism of compactification the mapping $M^{11} \rightarrow M^{4} \times S^{7}$ is made via the Kaluza-Klein formalism which mathematically can be understood in terms of fiber bundles.

The Fano matroid $F_{7}$ is one of the most important on matroid theory essentially because, among other things, it is the only minimal binary irregular matroid. The ground set for $F_{7}$ is

$$
E=\{1,2,3,4,5,6,7\} .
$$

The bases of $F_{7}$ are all those subsets of $E$ with three elements except the seven subsets $f_{1}=\{1,2,3\}, f_{2}=\{5,1,6\}, f_{3}=\{6,4,2\}, f_{4}=\{4,3,5\}$, $f_{5}=\{4,7,1\}, f_{6}=\{6,7,3\}$ and $f_{7}=\{5,7,2\}$. The dual $F_{7}^{*}$ of $F_{7}$ can be obtained from the complements of the bases of $F_{7}$.

In order to link $F_{7}$ with octonions let us introduce the set

$$
\mathcal{E}=\left\{e_{1}, e_{2}, e_{3}, e_{4}, e_{5}, e_{6}, e_{7}\right\} .
$$

We shall denote by $p_{1}=\left\{e_{1}, e_{2}, e_{3}\right\}, p_{2}=\left\{e_{5}, e_{1}, e_{6}\right\}, p_{3}=\left\{e_{6}, e_{4}, e_{2}\right\}$, $p_{4}=\left\{e_{4}, e_{3}, e_{5}\right\}, p_{5}=\left\{e_{4}, e_{7}, e_{1}\right\}, p_{6}=\left\{e_{6}, e_{7}, e_{3}\right\}$ and $p_{7}=\left\{e_{5}, e_{7}, e_{2}\right\}$ the corresponding subsets $f_{i}$, with $i=1,2, \ldots 7$. Let us write an octonion in the form

$$
q=q_{0} e_{0}+q_{1} e_{1}+q_{2} e_{2}+q_{3} e_{3}+q_{4} e_{4}+q_{5} e_{5}+q_{6} e_{6}+q_{7} e_{7},
$$

where $e_{0}$ denotes the identity. The imaginary units $e_{i}$ determine the product of octonions through the formula

$$
e_{i} e_{j}=-\delta_{i j}+\psi_{i j}^{k} e_{k}
$$

where $\delta_{i j}$ is the Kronecker delta and $\psi_{i j k}=\psi_{i j}^{l} \delta_{l k}$ are the fully antisymmetric structure constants. By taking the $\psi_{i j k}$ equal to 1 or -1 for each of the seven combinations $p_{i}$ we can obtain every value of $\psi_{i j k}$.

The basic associator of any three imaginary units $e_{i}, e_{j}$ and $e_{k}$ is

$$
\left\langle e_{i}, e_{j}, e_{k}\right\rangle=\left(e_{i} e_{j}\right) e_{k}-e_{i}\left(e_{j} e_{k}\right)=\varphi_{i j k m} e_{m},
$$

where $\varphi_{i j k l}$ is a fully antisymmetric four index tensor. This means that the octonion (Cayley) algebra is not associative and in fact it is an alternative 
algebra. It turns out that $\varphi_{i j k l}$ and $\psi_{i j k}$ are dual to each other. Specifically, they are related by

$$
\varphi_{i j k l}=(1 / 3 !) \epsilon_{i j k l m n r} \psi_{m n r}
$$

where $\epsilon_{i j k l m n r}$ is the fully antisymmetric Levi-Civita tensor, with $\epsilon_{12 \ldots 7}=1$.

Anticipating a connection between $F_{7}$ and octonions we observe that giving the numerical values $f_{i}$ for the indices of $\psi_{m n r}$ and using (13) we get the other seven subsets of $E$ with four elements used to define $F_{7}^{*}$. For instance, if we consider $f_{1}$ then we have $\psi_{123}$ and (13) gives $\varphi_{4567}$ which leads to the circuit subset $\{4,5,6,7\}$ of $F_{7}^{*}$.

Now, consider the matrix

$$
A=\left(\begin{array}{lllllll}
1 & 0 & 1 & 0 & 1 & 0 & 1 \\
1 & 1 & 0 & 0 & 0 & 1 & 1 \\
0 & 1 & 1 & 1 & 0 & 0 & 1
\end{array}\right)
$$

If we denote by $v_{i}$ the columns of this matrix, it is found that the bases of the matrix $A$ are those subsets with three columns except the subsets $h_{1}=\left\{v_{1}, v_{2}, v_{3}\right\}, h_{2}=\left\{v_{5}, v_{1}, v_{6}\right\}, h_{3}=\left\{v_{6}, v_{2}, v_{4}\right\}, h_{4}=\left\{v_{4}, v_{3}, v_{5}\right\}, h_{5}=$ $\left\{v_{4}, v_{7}, v_{1}\right\}, h_{6}=\left\{v_{6}, v_{7}, v_{3}\right\}$ and $h_{7}=\left\{v_{5}, v_{7}, v_{2}\right\}$. This shows that $F_{7}$ is not only realizable by the matrix $A$ but also that it is a binary matroid. Actually, we have performed the map $\varphi(i)=v_{i}$ and consequently the ground set $\{1,2,3,4,5,6,7\}$ becomes $\left\{v_{1}, v_{2}, v_{3}, v_{4}, v_{5}, v_{6}, v_{7}\right\}$ and the subsets $f_{i}$ are mapped to $h_{i}$

In principle, we can associate the chirotope

$$
\chi\left(i_{1}, i_{2}, i_{3}\right)=\operatorname{sign} \operatorname{det}\left(v_{i_{1}}, v_{i_{2}}, v_{i_{3}}\right)
$$

to $F_{7}$. But one of the intriguing properties of $F_{7}$ is that it is not orientable matroid. Without formally proving this fact (see Ref. 9 for a formal proof), one can verify by hand that $\chi\left(i_{1}, i_{2}, i_{3}\right)$ does not satisfy property $(\chi i i i)$. Anyhow, a relationship between $\chi\left(i_{1}, i_{2}, i_{3}\right)$ and the structure constants for octonions $\psi_{i_{1} i_{2} i_{3}}$ can be accomplished by means of the formula

$$
\psi_{i_{1} i_{2} i_{3}}+\chi\left(i_{1}, i_{2}, i_{3}\right)=C_{i_{1} i_{2} i_{3}}
$$

where $C_{i_{1} i_{2} i_{3}} \in\{-1,1\}$ can be identified with the chirotope of the uniform matroid $U_{3,7}$ which is an excluded minor for $G F(5)$-representability, where $G F(q)$ denotes a finite field of order $q$. The expression (16) shows that $\psi_{i_{1} i_{2} i_{3}}$ 
and $\chi\left(i_{1}, i_{2}, i_{3}\right)$ determine $C_{i_{1} i_{2} i_{3}}$. This means that $F_{7}$ and the octonions are complementary concepts of the oriented uniform matroid $\mathcal{M}\left(U_{3,7}\right)$ structure.

In order to understand further the formula (16) let us compute $\chi\left(i_{1}, i_{2}, i_{3}\right)$ for the different values of the indices $i_{p}$. Since the different $h_{i}$ correspond to linear dependent vector columns of $A$ we find that the chirotope $\chi\left(i_{1}, i_{2}, i_{3}\right)$ vanishes for the different $h_{i}$. Hence, using (15) we find that the only nonvanishing terms of $\chi\left(i_{1}, i_{2}, i_{3}\right)$ are

$$
\begin{array}{lllllll}
124+ & 125+ & 126- & 127+ & 134- & 135+ & 136- \\
137- & 145+ & 146- & 157- & 167+ & 234- & 235+ \\
236+ & 237+ & 245+ & 247+ & 256+ & 267- & 346- \\
347- & 356+ & 357+ & 456+ & 457+ & 467- & 567+
\end{array}
$$

On the other hand, for the different non-zero values of the octonions structure constants $\psi_{i_{1} i_{2} i_{3}}$, we can choose any consistent sign values for the different $h_{i}$. We shall make the following choice

$$
123+147-156-246+257-345-367-.
$$

We observe that $\chi$ takes sign values different from zero precisely when the corresponding $\psi$ takes sign values equal to zero and vice versa. Therefore, this is another way of showing that $\chi$ and $\psi$ are complementary concepts.

Now if we put together the sign set combinations (17) and (18) according to (16) we get the sign values for $C_{i_{1} i_{2} i_{3}} \in\{-1,1\}$ which can be identified with the chirotope of the oriented uniform matroid $\mathcal{M}\left(U_{3,7}\right)$. From (17) we can check by a direct computation that $F_{7}$ is not orientable, while from (17) and (18) we can verify the orientability of $U_{3,7}$.

How is the above matroid-octonions scenario related to $G_{2}$-holonomy of $D=11$ supergravity? As it is known recent investigations of M-theory have suggested to take $G_{2}$-holonomy seriously for the $7 D$ manifold of $D=11$ supergravity. In particular, by a generalization of the self-duality condition in four dimensions to eight dimensions Nishino and Rajpoot ${ }^{23}$ have shown a mechanism that implements the $G_{2}$-symmetry in supersymmetric models in $D \leq 8$. The interesting thing about this mechanism is that it opens the possibility of obtaining the supersymmetric vector multiplet with the full $S O(7)$ Lorentz covariance reduced to $G_{2}$ by dimensional reduction. The generalized self-duality condition used by Nishino and Rajpoot can be derived from the definitions 


$$
F_{[m n r 8]} \equiv \psi_{m n r}
$$

and

$$
F_{i j k l} \equiv \varphi_{i j k l},
$$

and by using (13). In fact, from (13), (19) and (20) it is found that $F_{\mu \nu \alpha \beta}$, with the Greek indices running from 1 to 8, satisfies the self-duality formula

$$
F^{\mu \nu \alpha \beta}=(1 / 4 !) \epsilon^{\mu \nu \alpha \beta \lambda \gamma \rho \sigma} F_{\lambda \gamma \rho \sigma} .
$$

This extension of the octonionic relation (13) from $7 D$ to $8 D$ must have his corresponding extension in matroid theory in connection with $F_{7}$. In particular, this observation suggested the extension of the formula (16) to the form

$$
F_{\mu_{1} \mu_{2} \mu_{3} \mu_{4}}+\chi\left(\mu_{1}, \mu_{2}, \mu_{3}, \mu_{4}\right)=C_{\mu_{1} \mu_{2} \mu_{3} \mu_{4}} .
$$

Our task is now to find now the mathematical meaning of $\chi\left(\mu_{1}, \mu_{2}, \mu_{3}, \mu_{4}\right)$ and $C_{\mu_{1} \mu_{2} \mu_{3} \mu_{4}}$. First of all, since the chirotopes are defined in terms of the bases of a matroid, it follows that $\chi\left(\mu_{1}, \mu_{2}, \mu_{3}, \mu_{4}\right)$ should correspond to a four-rank matroid. Further, $\chi\left(\mu_{1}, \mu_{2}, \mu_{3}, \mu_{4}\right)$ must have the property to be reduced to the chirotope $\chi\left(i_{1}, i_{2}, i_{3}\right)$ associated to $F_{7}$. This means that such a four-rank matroid should provide an extension of $F_{7}$. In other words $F_{7}$ should be a minor of such a four-rank matroid. Moreover, the self-duality of $F_{\mu_{1} \mu_{2} \mu_{3} \mu_{4}}$ should imply a self-duality not only for $\chi\left(\mu_{1}, \mu_{2}, \mu_{3}, \mu_{4}\right)$ but also for $C_{\mu_{1} \mu_{2} \mu_{3} \mu_{4}}$. This means that the four-rank matroid must be a self-dual matroid. Happily, there is a four rank matroid satisfying all these requirements. In fact, the affine matroid $A G(3,2)$ is a self-dual four-rank matroid and among its minors it has the matroid $F_{7}$. Specifically, $A G(3,2)$ is not graphic, nor cographic, and every single-element deletion is isomorphic to $F_{7}^{*}$ and every single-element contraction is isomorphic to $F_{7}$. Furthermore, one finds that the chirotope $\chi\left(\mu_{1}, \mu_{2}, \mu_{3}, \mu_{4}\right)$ associated to $A G(3,2)$ is self dual and has all the desired properties.

In connection with $C_{\mu_{1} \mu_{2} \mu_{3} \mu_{4}}$, we already mentioned that this object should be self-dual. It must also correspond to the chirotope of a four rank matroid. Since $F_{\mu_{1} \mu_{2} \mu_{3} \mu_{4}} \neq 0$ when $\chi\left(\mu_{1}, \mu_{2}, \mu_{3}, \mu_{4}\right)=0$ and $F_{\mu_{1} \mu_{2} \mu_{3} \mu_{4}}=0$ when $\chi\left(\mu_{1}, \mu_{2}, \mu_{3}, \mu_{4}\right) \neq 0$ we find that $C_{\mu_{1} \mu_{2} \mu_{3} \mu_{4}}$ should be associated to a four-rank matroid with an underlying set $E=\{1,2, \ldots, 8\}$ and with its bases given by all of the four element subsets of $E$. The candidate is, of course, 
the uniform matroid $U_{4,8}$ which is orientable. In general, the uniform matroid $U_{p, q}$ has the self dual property $U_{p-q, q}=U_{p, q}$ and therefore we discover that $U_{4,8}$ is also self-dual as required.

\section{GRAVITOID THEORY}

A physical theory that combines the concepts of matroid and graviton we shall call it gravitoid theory. From this definition it turns out that the connection of the Fano matroid $F_{7}$ and the affine matroid $A G(3,2)$ with $D=11$ supergravity discussed in the previous section can be considered as part of the gravitoid theory.

Perhaps the simplest motivation to be interested in gravitoid theory may follow from the heuristic observation that since the graviton can be represented classically as a symmetric second-rank matrix tensor and a matroid is a more general concept than a matrix one should expect that a gravitonmatroid connection may lead to an extension of the concept of the graviton. (It seems to be reasonable to call such a generalized gravitational structure the gravitoid.) The real motivation, however, may arise from the idea of establishing a duality principle in M-theory. As we mentioned in the introduction, duality is one of the most attractive features in matroid theory. In a certain sense, matroid theory is the theory of duality. Therefore, matroid theory offers an excellent mathematical framework to incorporate a duality principle in M-theory. It turns out that although the gravitoid theory may be interesting by itself, it can also teach us, among other things, how to achieve such a quest.

Before we discuss the formal expected approach for the gravitoid theory, it is first convenient to briefly mention the recently proposed mathematical formalism of matroid bundle. This mathematical structure is the combinatorial analogue of real vector bundles in which a "base space" is a simplicial complex and the "fibers" are oriented matroids. The idea of matroid bundles arose from the concept of combinatorial differential manifolds introduced by MacPherson ${ }^{14}$ in 1993 and evolved very rapidly on several fronts. In particular, a close connection between matroid bundle theory and characteristic classes has emerged. ${ }^{24,25}$

Two main ideas make the matroid bundle structure possible: oriented matroids and simplicial complexes. In order to define formally matroid bundles we closely follow Anderson's work (see Ref. 15). Let $\xi: \mathcal{E} \rightarrow B$ be a real 
vector bundle, where $B$ is a $n$-dimensional base space. We assume $B$ to be a triangulable compact space, i.e., $B$ has an associated simplicial complex. For each point $b$ of $B$ the vectors $\left\{e_{1}(b), \ldots, e_{n}(b)\right\}$ span the space $\xi^{-1}(b)$. We can associate an oriented matroid with underlaying set $E=\{1,2, \ldots, n\}$ to the vectors $\left\{e_{1}(b), \ldots, e_{n}(b)\right\}$.

A rank- $k$ matroid bundle is a pair $\left(S_{B}, \mathcal{M}\right)$ where $S_{B}$ is a partially ordered set and $\mathcal{M}$ is a rank- $k$ oriented matroid associated to each element $b$ so that $\mathcal{M}(b)$ weakly maps to $\mathcal{M}\left(b^{\prime}\right)$ whenever $b \geq b^{\prime}$. Intuitively, and in analogy to the case of a real vector bundle, $S_{B}$ corresponds to the base space $B$ and the oriented matroid $\mathcal{M}$ to the fiber of the bundle $\mathcal{E}$ (For a complete exposition see Ref. 15).

In particular, in the case of tangent matroid bundles one associates an oriented matroid to each tangent space and one realizes a differentiable manifold by a combinatorial differential manifold. In what follows, we shall focus our attention on this special kind of matroid bundle.

In order to introduce the gravitoid concept it is first convenient to recall, in brief, how the metric is introduced in a tangent bundle. Consider a tangent bundle over the differentiable manifold $B$ :

$$
T(B)=\bigcup_{b \in B} T_{b}(B) .
$$

Here, the tangent space $T_{b}(B)$ plays the role of the fiber of $T(B)$. (We assume that $B$ is a triangulable manifold.) Of course, $T_{b}(B)$ is a vector space and consequently $T(B)$ provides an example of a real vector bundle. The dual of $T(B)$ is the cotangent bundle $T^{*}(B)$ defined in the form

$$
T^{*}(B)=\bigcup_{b \in B} T_{b}^{*}(B)
$$

where $T_{b}^{*}(B)$ is the dual vector space of $T_{b}(B)$. Thus, whereas $T_{b}(B)$ is a vector space of contravariant vectors, $T_{b}^{*}(B)$ is a vector space of covariant vectors (1-forms). Moreover, it can be proved that the dimension of $T_{b}^{*}(B)$ is equal to the dimension of $T_{b}(B)$.

Take $\frac{\partial}{\partial b^{\mu}}(1 \leq \mu \leq n)$ as the base vectors of $T_{b}(B)$ and $d b^{\mu}$ as the corresponding bases (one forms) of $T_{b}^{*}(B)$. Consider a vector $V^{\mu} \frac{\partial}{\partial b^{\mu}}$ and its dual one-form $V_{\mu} d b^{\mu}$. The tangent space isomorphism

$$
g: T_{b}(B) \rightarrow T_{b}^{*}(B)
$$


has the component representation

$$
V_{\mu}=g_{\mu \nu} V^{\nu} .
$$

Expressions $(25)$ or $(26)$ can be used to define an inner product $\langle\omega, V\rangle$ : $T_{b}^{*}(B) \otimes T_{b}(B) \rightarrow R$, where $R$ is the set of real numbers. In component representation this leads to

$$
\langle\omega, V\rangle=\omega_{\mu} V^{\mu},
$$

which by virtue of (26) can also be written as

$$
\langle\omega, V\rangle=g_{\mu \nu} \omega^{\mu} V^{\nu}
$$

Usually the matrix $g_{\mu \nu}$ is called a metric if it is symmetric and positive definite, so $\langle V, V\rangle$ has the meaning of square norm of $V$. However, in the case of a pseudo Riemannian space the positive definite condition can be dropped and we can still call the matrix $g_{\mu \nu}$ a metric (or pseudometric). Moreover, in nonsymmetric gravitational theories the matrix $g_{\mu \nu}$ does not even satisfy the symmetric condition. Therefore, in order to be as general as possible we shall call a metric just the isomorphism (25), without specifying any additional condition.

Let us now return to our goal of finding a mathematical structure for the gravitoid system. In the case of a tangent matroid bundle, $T_{b}(B)$ is replaced by an oriented matroid $\mathcal{M}_{T_{b}}$ and the base space is replaced by a simplicial complex $S_{B}$. Just like one can associate to $T_{b}(B)$ the dual tangent space $T_{b}^{*}(B)$, one can associate to $\mathcal{M}_{T_{b}}$ the dual oriented matroid $\mathcal{M}_{T_{b}}^{*}$. Thus, one should expect that the isomorphism $g: T_{b}(B) \rightarrow T_{b}^{*}(B)$ leads to the corresponding isomorphism

$$
\mathcal{G}_{g}: \mathcal{M}_{T_{b}} \rightarrow \mathcal{M}_{T_{b}}^{*}
$$

In general if an oriented matroid is realizable, its dual is also realizable. Therefore, if $\mathcal{M}_{T_{b}}$ is realizable then $\mathcal{M}_{T_{b}}^{*}$ is also realizable. Let us denote by $A$ the $k \times n$ matrix over a field $R$ representing the vector matroid $\mathcal{M}_{T_{b}}[A]$. The ground set $E$ of $\mathcal{M}_{T_{b}}[A]$ are the columns of $A$. Similarly let us denote by $A^{*}$ the corresponding matrix associated to the dual vector matroid $\mathcal{M}_{T_{b}}^{*}[A]$. In this context the map (29) has the component representation

$$
a_{i}=\mathcal{G}_{i j} a^{j}
$$


where $a^{j} \in A$ and $a_{i} \in A^{*}$.

Now, the question arises whether there is a relation between the two maps $g$ and $\mathcal{G}$. The map $\mathcal{G}$ can be understood as the combinatorial analogue of the metric $g$. For this reason we shall call $\mathcal{G}_{i j}$ the matroid metric. Since the simplicial complex $S_{B}$ is homeomorphic to the smooth manifold $B$, i.e., $\lambda$ : $S_{B} \rightarrow B$ is a homeomorphism, we have the induced map $\eta: \mathcal{M}_{T_{b}} \rightarrow V_{b}(B)$, where $V_{b}(B)$ is a finite set of vectors in $T_{b}(B)$. Hence, (25) and (29) imply that there must exist a map $\eta^{*}: \mathcal{M}_{T_{b}}^{*} \rightarrow V_{b}^{*}(B)$, where $V_{b}^{*}(B)$ is a finite set of covectors in $T_{b}^{*}(B)$ and $\eta^{*}=g \eta \mathcal{G}^{-1}$. This result establishes the connection between $g$ and $\mathcal{G}$.

Our next step is to associate a gravitational theory to the matroid metric $\mathcal{G}_{i j}$. For this purpose we first need to introduce the combinatorial analogue of a connection and curvature. At this respect we shall relay on Ref. 24. The idea is to define 1-cocycle $\Theta$ and 2-cocycle $\rho^{*} \Omega=\delta \Theta$ on each local system on $S_{B}$. It is worth mentioning that, in order to find a combinatorial formula for the Pontrjagin classes, Gelfand and MacPerson consider $\Theta$ and $\Omega$ as the combinatorial analogue of connection and curvature of $B$, respectively.

The quest now is to find a relation between $\mathcal{G}$ and $\Omega$. In principle, we can achieve this by applying a Palatini method to certain proposed action $I=I(\mathcal{G}, \Omega)$. The question is, therefore, what is the form of $I(\mathcal{G}, \Omega)$ ? A partial answer to this question may come from the link between matroid theory and Chern-Simons theory found in Ref. 7. For this reason let us briefly review the main ideas of Ref. 7 .

It is known that if we choose $B^{3}=S^{3}, G=S U(2)$ and $\rho_{r}=C^{2}$ for all the link components then the Witten's partition function

$$
Z(L, k)=\int D A \exp \left(S_{c s}\right) \prod_{r=1}^{n} W\left(L_{r}, \rho_{r}\right),
$$

where $S_{C S}$ is the Chern-Simons action

$$
S_{C S}=\frac{k}{2 \pi} \int_{M^{3}} \operatorname{Tr}\left(A \wedge d A+\frac{2}{3} A \wedge A \wedge A\right)
$$

and $W\left(C_{i}, \rho_{i}\right)$ is the Wilson line

$$
W\left(L_{r}, \rho_{r}\right)=\operatorname{Tr}_{\rho_{r}} P \exp \left(\int_{L_{r}} A_{i}^{a} T_{a} d x^{i}\right)
$$

reproduces the Jones polynomial 


$$
Z(L, k)=V_{L}(t) .
$$

Here, $A=A_{i}^{a} T_{a} d x^{i}$, with $T_{a}$ the generators of the Lie algebra of a gauge group $G$ and the symbol $P$ means the path-ordering along the knots $L_{r}$. The parameter $t$ is

$$
t=e^{\frac{2 \pi i}{k}}
$$

and $V_{L}(t)$ denotes the Jones polynomial satisfying the skein relation;

$$
t^{-1} V_{L_{+}}-t V_{L_{-}}=\left(\sqrt{t}-\frac{1}{\sqrt{t}}\right) V_{L_{0}},
$$

where $L_{+}, L_{-}$and $L_{0}$ are the standard notation for overcrossing, undercrossing and zero crossing.

On the other hand, Thistlethwaite ${ }^{26}$ showed that if $L$ is an alternating link and $G(L)$ the corresponding planar graph, then the Jones polynomial $V_{L}(t)$ is equal to the Tutte polynomial $T_{G}\left(-t,-t^{-1}\right)$ up to a sign and a factor power of $t$. Specifically, we have

$$
V_{L}(t)=\left(-t^{\frac{3}{4}}\right)^{w(L)} t^{\frac{-(r-n)}{4}} T_{G}\left(-t,-t^{-1}\right),
$$

where $w(L)$ is the writhe and $r$ and $n$ are the rank and the nullity of $G$, respectively. Here, $V_{L}(t)$ is the Jones polynomial of the alternating link $L$. The Tutte polynomial associated to each graph $G$ is a polynomial $T_{G}\left(x, x^{-1}\right)$ with the property that if $G$ is composed solely of isthmus and loops then $T_{G}\left(x, x^{-1}\right)=x^{I} x^{-l}$, where $I$ is the number of isthmuses and $l$ is the number of loops. The polynomial $T_{G}$ satisfies the skein relation

$$
T_{G}=T_{G^{\prime}}+T_{G^{\prime \prime}},
$$

where $G^{\prime}$ and $G^{\prime \prime}$ are obtained by deleting and contracting respectively an edge that is neither a loop nor an isthmus of $G$.

A theorem due to Tutte allows to compute $T_{G}\left(-t,-t^{-1}\right)$ from the maximal trees of $G$. In fact, Tutte proved that if $\mathcal{B}$ denotes the set of maximal trees in a graph $G, i(\mathcal{B})$ denotes the number of internally active edges in $G$, and $e(B)$ refers to the number of the externally active edges in $G$ (with respect to a given maximal tree $B \in \mathcal{B}$ ) then the Tutte polynomial is given by the formula

$$
T_{G}\left(-t,-t^{-1}\right)=\sum_{B \subseteq \mathcal{B}} x^{i(B)} x^{-e(B)},
$$


where the sum is over all elements of $\mathcal{B}$. It is worth mentioning that the Tutte polynomial $T_{G}\left(-t,-t^{-1}\right)$ computed according to (39) uses the concept of a graphic matroid $M(G)$ defined as the pair $(E, \mathcal{B})$, where $E$ is the set of edges of $G$.

Therefore, we have the connections $M(G) \leftrightarrow T_{G}\left(-t,-t^{-1}\right) \leftrightarrow V_{L}(t) \leftrightarrow$ $Z(L, k)$. These bridges allow to transfer information from $M(G)$ to $Z(L, k)$ and conversely. In particular, if we change the notation $T_{G}\left(-t,-t^{-1}\right) \rightarrow$ $T_{M(G)}(t)$ and $Z(L, k) \rightarrow Z_{M(G)}(k)$ one discovers that the duality property of the Tutte polynomial

$$
T_{G}\left(-t,-t^{-1}\right)=T_{G^{*}}\left(-t^{-1},-t\right)
$$

can be expressed as

$$
T_{M(G)}(t)=T_{M^{*}(G)}\left(t^{-1}\right)
$$

and consequently from (34) and (37) we find that the partition function $Z_{M(G)}(k)$ gets the dual property

$$
Z_{M(G)}(k)=Z_{M^{*}(G)}(-k),
$$

(see Ref. 7 for details). This expression is of special relevance because it is exactly the kind of symmetry that one may expect in M-theory. Writing $k=\ln \tau$, where $\tau$ is a constant parameter (perhaps complex) one finds that the symmetry (42) in $Z_{M(G)}(k)$ is related to the S-duality symmetry $\tau \rightarrow-\frac{1}{\tau}$ which is one of the key symmetries linking different superstring theories.

In the gravitoid theory one should expect similar, but not quite the same construction as the matroid-Chern-Simons approach. First of all, the ChernSimons action in (31) is not written in abstract combinatorial terms. (Perhaps, here again the Gelfand-MacPerson ${ }^{24}$ procedure could be useful.) Secondly, the matroid theory enters in the scenario via graph theory. But graph theory is just an example of the simplicial complex formalism, so one needs the analogue for the Tutte polynomial for higher dimensional simplexes and, as far as we know, no such a mathematical structure is available in the mathematical literature. Nevertheless, let us just simply write the expected abstract symmetry that the gravitoid partition function should have, namely

$$
Z_{\mathcal{M}_{T_{b}}}(\mathcal{G}, \Omega)=Z_{\mathcal{M}_{T_{b}}^{*}}\left(\mathcal{G}^{*}, \Omega^{*}\right),
$$

where $\Omega^{*}$ is the 2 -cocycle associated to $\mathcal{G}^{*}$. Since every matroid $\mathcal{M}$ satisfies 


$$
\mathcal{M}^{* *}=\mathcal{M}
$$

we should have $\mathcal{G}^{*}=\mathcal{G}^{-1}$. The fact that any matroid has its corresponding dual matroid is translated as a dual symmetry of the partition function $Z_{\mathcal{M}_{T_{b}}}(\mathcal{G}, \Omega)$. This is a remarkable consequence of using matroid theory as a mathematical framework. In order to have a complete picture in the expression (43) it is necessary to find the combinatorial analogue of the concept of classical action. Although this seems to be not a trivial problem, we can speculate that the answer should be something similar to the combinatorial formula for the Pontrjagin classes due to Gelfand and MacPherson. ${ }^{24}$ Oriented matroids and the simplicial complex formalism allowed these authors to find the combinatorial analogue of the Chern-Weil formula

$$
\tilde{p}_{i}(E)=(-1)^{i} \pi_{\star} \Omega^{(e-2+2 i)} .
$$

Here, $\pi_{\star}$ represents an integration over a fiber. In Chern-Weil theory the curvature is defined by $\rho^{\star} \Omega=d \Theta$, where $\Theta$ is a one form induced by the connection on the bundle $E$. In (45) $e$ is the fiber dimension of $E$. The combinatorial analogue of (45) proposed by Gelfand and MacPherson is

$$
\left.\tilde{p}_{i}(X) \frown[X]=(-1)^{i} \pi_{\star}\left(\frac{1}{2} \Omega\right)^{(n-2+2 i)} \frown \phi\right),
$$

where $\phi$ is a fixing cycle for the simplicial complex $X$ (For details and further information we refer the reader to the original Ref. 24 of Gelfand and MacPherson.)

Since in matroid theory we have that any two connected matroids $\mathcal{M}_{1}$ and $\mathcal{M}_{2}$ satisfy the expression $\left(\mathcal{M}_{1} \oplus \mathcal{M}_{2}\right)^{*}=\mathcal{M}_{1}^{*} \oplus \mathcal{M}_{2}^{*}$ we discover the remarkable result that

$$
Z_{\mathcal{M}_{1 T_{b}}}=Z_{\mathcal{M}_{1 T_{b}}^{*}}
$$

and

$$
Z_{\mathcal{M}_{2 T_{b}}}=Z_{\mathcal{M}_{2 T_{b}}^{*}}
$$

if and only if

$$
Z_{\mathcal{M}_{1 T_{b}} \oplus \mathcal{M}_{2 T_{b}}}=Z_{\left(\mathcal{M}_{1 T_{b}} \oplus \mathcal{M}_{2 T_{b}}\right)^{*}}
$$


Besides its importance in gravitoid theory, the equation (43) may motivate a similar construction for M-theory. As it was mentioned before the five known superstring theories in $9+1$ dimensions can be understood via duality as a different vacuum of the M-theory. Therefore, one should expect that the partition function associated to M-theory should be invariant under certain duality symmetry such as (42) or (43).

\section{COMMENTS}

Matroid bundle is a remarkable mathematical structure that promises many interesting applications not only in mathematics but also in physics. In the present work we have shown the possibility of using matroid bundle as a framework for a new gravitational theory called gravitoid theory. The essential idea in this theory is to combine the concepts of graviton and matroid. One of the hopes is that gravitoid theory shows us how to develop M-theory from M(atroid)-theory. In essence we have proposed a partition function with a duality symmetry for the gravitoid system which may suggest a similar structure for M-theory.

Our procedure was focused on realizable tangent matroid bundles. However, since not all of the oriented matroids are realizable there must be more general tangent matroid bundles and therefore a more general gravitoid theory. Mathematically, a similar question has been considered in Ref. 14 where it was raised the question whether all combinatorial differential manifolds are topological manifolds.

One of the attractive features of matroid bundle is that it offers the possibility of using new combinatorial techniques to study characteristic classes. In particular, it has been proved that matroid bundles may be the basis for new studies on characteristic classes of differential manifolds. Exam-

ples of this fact is the Gelfand-MacPherson's combinatorial formula ${ }^{24}$ for the Pontrjagin classes and the Anderson-Davis's combinatorialization ${ }^{25}$ of the Stiefel-Whitney classes and Euler classes. These characteristic classes play an essential role in theoretical physics. For instance, Stiefel-Whitney classes are useful to determine the spin structure of a smooth manifold and Euler classes and Pontrjagin classes are useful topological invariants in the formulation of the MacDowell-Mansouri's formalism (see Refs. 27 and references there in) in supergravity. These observations suggest and motivate the search of a link between the gravitoid theory and these combinatorial characteristic 
classes.

Gravitoid theory can be understood as the combinatorialization of the bosonic sector of supergravity in any dimension and in particular in $D=11$. In view of the MacPherson's conjecture ${ }^{14,16}$ that all characteristic classes of a vector bundle should have their corresponding characteristic classes in matroid bundle one should expect combinatorialization of Chern-Simons theory and topological gravity ${ }^{28}$. From this perspective it is tempting to speculate about the possibility that gravitoid theory may contain, in fact, the combinatorialization of topological gravity.

There are many other open questions. Is gravitoid theory an alternative for quantum gravity? What is the supersymmetrization of the gravitoid theory? What is the precise relation between the gravitoid theory and $D=11$ supergravity? It seems that the combinatorialization of the Pontrjagin classes implies an exotic combinatorial $7 D$ sphere. Is the gravitoid theory related to this exotic structure via $D=11$ supergravity? Our hope is that the present work stimulates a research to answer these and other related questions.

Acknowledgment: We would like to thank C. Austin for calling our attention about the Anderson and Davis's references. We also like to thank to J. Saucedo and G. Compoy for helpful comments.

\section{References}

[1] P. K. Townsend, "Four lectures on M-theory," Proceedings of the ICTP on the Summer School on High Energy Physics and Cosmology, June 1996, hep-th/9612121.

[2] M. J. Duff, Int. J. Mod. Phys. A 11, 5623 (1996), hep-th/9608117.

[3] P. Horava and E. Witten, Nucl. Phys. B 460, 506 (1996).

[4] M. Green, V. Schwarz and E. Witten, Superstrings Theory (Cambridge University Press, Cambridge, 1987) Vol I and II; M. Kaku, Introduction to Superstrings (Spring-Verlag, Berlin, 1990).

[5] H. Whitney, Am. J. Math. 57, 509 (1935).

[6] J. A. Nieto, Rev. Mex. Fis. 44, 358 (1998). 
[7] J. A. Nieto and M. C. Marin, J. Math. Phys. 41, 7997 (2000).

[8] J. A. Nieto, "Searching for a connection between matroid theory and string theory," hep-th/0212100.

[9] J. G. Oxley, Martroid Theory, (Oxford University Press, New York, 1992)

[10] D. J. A. Welsh, Martroid Theory, (Academic, London, 1976).

[11] R. J. Wilson, Introduction to Graph Theory, 3rd ed. (Wiley, New York, 1895).

[12] J. P. S. Kung, A Source Book in Matroid Theory, (Birkhauser, Boston, 1986).

[13] K. Ribnikov, Análisis Combinatorio, (Editorial Mir, Moscú, 1988)

[14] R. D. Macpherson, "Combinatorial differential manifolds: a symposium in honor of John Milnor's sixtieth birthay," pp. 203-221 in Topological methods on modern mathematics (Stony Brook, NY, 1991), edited by L. H. Goldberg and A. Phillips, Houston, 1993.

[15] L. Anderson, New Perspectives. in Geom. Comb. 38, 1 (1999).

[16] D. Biss, "Some applications of oriented matroids to topology," $\mathrm{PhD}$. thesis, MIT, 2002.

[17] Babson, "A combinatorial flag space," PhD. thesis, MIT, (1993).

[18] A. Bjorner, M. Las Verganas, N. White and G. M. Ziegler, Oriented Martroids, (Cambridge University Press, Cambridge, 1993).

[19] H. Whitney, Trans. Amer. J. Math. 34, 339 (1932)

[20] L. Gutierrez-Novoa, Pacific J. Math. 15, 1337 (1965).

[21] F. Englert, Phys. Lett. B 119, 339 (1982); F. Gursey and C. Tze, Phys. Lett. B 127, 191 (1983).

[22] I. L. Kantor and A.S. Solodovnikov, Hypercomplex Numbers; An Elementary Introduction to Algebras, (Spring Verlag, New York, 1989); J. C. Baez, Bull. Amer. Math. Soc. 39, 145 (2002). 
[23] H. Nishino and S. Rajpoot, "Octonions, G(2) symmetry, generalized selfduality and supersymmetries in dimensions D less than or equal 8," hep-th/0210132.

[24] I. M. Gelfand and R. D. Macpherson, Bull. Amer. Math. Soc. 26, 304 (1992).

[25] L. Anderson and J. F. Davis, "Mod 2 Cohomolgy of Combinatorial Grassmannians," math.GT/9911158.

[26] M. Thistlethwaite, Topology 26, 297 (1987).

[27] H. Garcia-Compean, J. A. Nieto, O. Obregon and C. Ramirez, Phys. Rev. D 59, 124003 (1999); J. A. Nieto and J. Socorro Phys. Rev. D 59, 041501 (1999); J. A. Nieto, J. Socorro and O. Obregon, Phys. Rev. Lett.76, 3482 (1996).

[28] E. Witten, Phys. Lett. B 206, 601 (1988). 